\title{
Psychophysical correlates of global motion processing in the aging visual system: A critical review
}

\author{
Claire V. Hutchinson ${ }^{1 *}$, Amanda Arena ${ }^{1}$, Harriet A. Allen ${ }^{2}$ and Tim Ledgeway ${ }^{2}$ \\ ${ }^{1}$ School of Psychology, College of Medicine, Biological Sciences and Psychology, \\ University of Leicester, Leicester, UK. \\ ${ }^{2}$ School of Psychology, University of Nottingham, Nottingham, UK. \\ Running title: Age and global motion
}

Keywords: age, global motion, random dot kinematograms

Correspondence:

Claire V. Hutchinson

School of Psychology

College of Medicine, Biological Sciences and Psychology

University of Leicester

Leicester

UK

LE1 9HN

ch190@1e.ac.uk

\begin{abstract}
The consequences of visual decline in aging have a fundamental and wide-reaching impact on age-related quality of life. It is of concern therefore that evidence suggests that normal aging is accompanied by impairments in the ability to effectively encode global motion. Global motion perception is a fundamentally important process. It enables us to determine the overall velocity of spatially-extensive objects in the world and provides us with information about our own body movements. Here, we review what is currently known about the effects of age on performance for encoding the global motion information available in random dot kinematograms (RDKs), a class of stimuli widely used to probe the mechanisms underlying motion perception. We conclude that age-related deficits in global motion perception are not all encompassing. Rather, they appear to be specific to certain stimulus conditions. We also examine evidence for an interaction between age and gender and consider the efficacy of techniques such as visual perceptual learning that may attenuate some of the visual deficits in the older adult population.
\end{abstract}

\section{Introduction}

Our ability to accurately perceive movement in the world around us requires a series of complex computations at multiple stages in our visual pathway. Our visual systems encode movement by comparing and decoding rapidly changing information that falls on our retinas. This information is drawn from, and influenced by, many sources, including information generated by the movement of objects in the world, by our own eye movements, and by our self-motion through space. Given the complexity of the information entering the visual system and its importance in everyday life, it is 
unsurprising that a considerable amount of the visual sensory cortex, including specialized areas in the extrastriate cortex, is involved in the registration and analysis of information concerning motion. Almost every daily activity is reliant upon our ability to accurately perceive motion, for example, navigating safely and effectively through the environment, judging the speed of oncoming traffic, even making a cup of tea. Indeed, there is evidence that patients with bilateral brain damage to motionsensitive brain areas are unable to carry out these tasks (Zihl, von Cramon \& Mai, 1983), the result of which is a debilitating impact on everyday quality of life. It is of great concern therefore that many studies report deficits in motion perception in the aged. This is compounded by evidence that poor vision is one of the leading causes of fall-related injury and injury-related death in the elderly (Harwood, 2001). In this review, we examine the effects of age on the ability to effectively encode global motion.

\section{Global motion}

Global motion refers to instances in which the individual trajectories of local moving elements in a visual scene come together to create a larger, more global, stimulus. Real world examples include the movement of a swarm of bees or a flock of birds, leaves blowing in a tree on a windy day or a rockslide. Other examples include the movement of ripples on the surface of water or gross movement of blades of grass or wheat in fields, all of which permit detection of wind direction. Although individual elements move along different local trajectories, their combined direction appears to move coherently. Global motion is commonly simulated in the laboratory using random dot kinematogram (RDK) patterns. An RDK is a motion stimulus made up of a number of individual, local dots. In a motion sequence some local dots move in the same direction (signal dots) whilst others move in random directions (noise dots). The greater the proportion of local elements that move in the same direction (i.e. the greater the motion coherence), the easier it becomes to discern the global motion. An example is given in Figure 1. Motion coherence thresholds correspond to the minimum number of local signal dots that have to move in the same direction to provide a reliable estimate of the global motion, i.e. the impression that the entire dot field is moving in a given direction. Human observers, and also non-human primates, are adept at encoding the direction of global motion. They typically only require a small percentage of local elements, often less than $5 \%$, to move in a given direction to be able to accurately determine the global direction of motion (e.g. Newsome \& Paré, 1988)

\section{The effects of age on translational global motion perception}

The preponderance of work investigating the effects of age on global motion perception has employed RDK patterns in which signal dots move along a translational trajectory (e.g. up vs. down or left vs. right). As a consequence, this section concentrates on age-related effects on translational global motion processing. The effects of age on other types of global motion will be discussed later in this review.

Many studies have shown an age-related decline in the perception of translational motion. Ball and Sekuler (1986) were among the first to use RDK stimuli to do so. Young (18 to 28 years) and old (62 to 72 years) participants were presented with a 
two-interval motion display in which the stimulus presented in one interval appeared in a different spatial location from the stimulus presented in the other interval. Participants were asked to identify whether the direction of motion in the two intervals had been the same or different. Older participants exhibited poorer performance on the task relative to the younger sample. Trick and Silverman (1991) compared global motion sensitivity in young adults, healthy older adults and older adults diagnosed with senile dementia Alzheimer's type (SDAT). The authors found that observers with SDAT had lower motion sensitivity than their healthy aged counterparts. In addition, healthy older adults exhibited impaired performance relative to young adults. Wojciechowski, Trick and Steinman (1995) compared younger (22.9 \pm 1.3 years $)$ and older ( $66.6 \pm 4.5$ years $)$ participants' global motion perception in central vision and 18 degrees in the nasal, temporal, superior and inferior peripheral visual fields. The results reveal impaired performance in old, compared to young individuals, at all visual field locations. Impairments were most marked in central vision. Tran, Silverman, Zimmerman and Feldon (1998) assessed the ability to judge the direction of translational RDK patterns in participants aged 19 to 92 years. In agreement with previous studies, they found an age-related deterioration in global motion processing. Billino, Bremmer and Gegenfurtner (2008) also found a deleterious effect of age on translational global motion perception in participants ranging from 20 to 82 years. However, the precise extent of age-related deficits are unresolved with estimates of deterioration between participants in their $20 \mathrm{~s}$ and $70 \mathrm{~s}$ ranging from around 2 to $13.5 \%$.

The findings of the studies outlined above have led to a consensus that translational global motion processing is impaired in the elderly. However, as research investigating the effects of normal aging on global motion perception has increased, a more complete picture has begun to emerge, one suggesting that, rather than being all encompassing, age-related effects are critically dependent on a number of specific factors.

Local dot speed appears to have a significant effect on the extent of age-related deficits in global motion perception. Using RDKs of different speeds ( 0.045 to 4 $\mathrm{deg} / \mathrm{sec}$ ), Snowden and Kavanagh (2006) found that age-related performance deficits were specific to local element speeds below $1 \mathrm{deg} / \mathrm{sec}$. At speeds above $1 \mathrm{deg} / \mathrm{sec}$, young and old participants performed equivalently. This parameter-dependent age effect has been supported by other studies. Allen, Hutchinson, Ledgeway and Gayle (2010) also failed to find an effect of age at dots speeds of $5.6 \mathrm{deg} / \mathrm{sec}$, at least when dot contrast was high. When dots travelled at $4.8 \mathrm{deg} / \mathrm{sec}$, Atchley and Anderson (1998) found minimal evidence of age-related differences in global motion processing. On the other hand, Tran et al. (1998) did find a marked effect of age when local dots moved at $2.5 \mathrm{deg} / \mathrm{sec}$. Although this finding appears to be at odds with others that used intermediate dot speeds, a speed of $2.5 \mathrm{deg} / \mathrm{sec}$ is relatively close to Snowden and Kavanagh's cut-off of $1 \mathrm{deg} / \mathrm{sec}$ and may reflect an effect of speed in combination with other factors, such as the magnitude of the dot displacement (jump size on each positional update), dot density, and image size. The picture is much clearer at relatively high dot speeds, where there is compelling evidence of an agerelated decline in motion sensitivity. At a dot speed of $22 \mathrm{deg} / \mathrm{sec}$, Atchley and Anderson (1998) found evidence of age-related decreases in performance in both central and peripheral $(<40 \mathrm{deg})$ vision. Other studies (Trick \& Siverman, 1991; Ball \& Sekuler, 1987; Wojciechowski et al., 1995; Billino et al. 2008) have also provided 
evidence of age-related global motion deficits when local dot speed was high, namely $6,10,28$ and 6.6 or $18.6 \mathrm{deg} / \mathrm{sec}$, respectively. These findings suggest that speedtuning changes with age and optimal sensitivity becomes restricted to a much narrower range of speeds in the aging visual system (see Table 1 for a synopsis of main findings).

Speed is not the only factor that heavily influences the extent of age-related deficits in global motion perception. There is also growing evidence that performance in the elderly may also be markedly influenced by the extent of the spatial displacement of local elements. Roudaia, Bennett, Sekuler and Pilz (2010) investigated the spatial and temporal integration limits of age-related motion processing deficits. They found that spatial displacement had a much greater adverse effect on performance in older adults than the length of the inter-stimulus-interval between frames (i.e. the time between when a dot disappears and reappears at a different spatial location). This was particularly evident at high displacements ( $>0.5 \mathrm{deg}$ on each positional update). Arena, Hutchinson \& Shimozaki (2011) have also shown that age-related deficits in global motion perception were more heavily mediated by dot spatial displacement magnitude than by speed. Complementary results have been shown by Andersen and $\mathrm{Ni}$ (2008) for shape perception based on kinetic occlusion. They found that agerelated impairments in the perception of shape were most heavily influenced by deficits in spatial rather than temporal integration. Indeed, the importance of integrating a motion signal over space is not restricted to the aged. Smith, Snowden and Milne (1994) for example have shown that while both spatial and temporal integration play a role in global motion perception, it is spatial integration that is fundamental to the processing of translational motion. It is not surprising therefore that age-related deficits in spatial integration have a marked effect on the ability to encode motion direction.

Dot visibility has also been found to influence performance on tasks that require global motion perception. Even in instances where there is a minimal effect of age, decreasing dot contrast has a more profoundly deleterious effect on performance levels of older participants. As mentioned previously, Allen et al. (2010) have shown that when high contrast dots move at $5.6 \mathrm{deg} / \mathrm{sec}$, older and younger adults' motion discrimination performance is comparable. However reducing dot contrast led to elevated motion coherence thresholds (impaired performance) for older, compared to younger adults. These findings demonstrate the important role of contrast encoding and sensitivity in global motion perception.

\section{Gradual decline or abrupt onset}

Many studies that have identified the existence of an age-related performance deficit in global motion perception have used discrete participant groups (i.e. categorised as either young or old), whereas others have addressed continuous performance changes throughout the adult lifespan. Where studies have addressed global motion processing across the lifespan, there is some disagreement regarding whether age-related deficits in global motion processing reflect a gradual decline throughout adulthood, or a sudden onset in old age, particularly in the 8th decade of life. Trick and Silverman (1991) for example, found an approximately linear, positive relationship between motion thresholds and age, estimating that an increase of around $1.36 \%$ coherence is required per decade in order to perceive global motion stimuli. Similarly, 
Wojciechowski et al. (1995) found performance on a direction-identification task decreased by $1.1 \%$ per decade. Other estimates include a $0.4 \%$ threshold increase per decade (Tran et al., 1998), and a $2.7 \%$ threshold increase per decade (Billino et al., 2008). The findings of other studies however suggest that the ability to encode global motion is relatively well preserved until around 70 years of age (Bennett, Sekuler \& Sekuler, 2007; Arena et al., 2011). It therefore currently remains unclear whether declines in global motion perception emerge gradually after adolescence or occur abruptly. Further investigation into the onset of such differences could provide insight into the neural underpinnings of these deficits.

\section{The influence of gender}

Another interesting issue that has emerged in the literature is the existence of genderspecific age deficits. Some studies have found that older women are particularly susceptible to age-related deficits in global motion perception, although the extent of this gender effect is presently unclear. For example, whilst Gilmore, Wenk, Naylor and Stuve (1992), found no overall differences between young (18 to 23 years) and old (62 to 83 years) observers' ability to encode global motion, they did find some indication of moderately impaired performance in older women. Tran et al. (1998) also noted lower performance levels in females however this trend did not reach statistical significance. The results of other studies suggest that, whilst older women do have significantly higher coherence thresholds (i.e. worse performance), this is also true of younger female participants (Billino et al., 2008), and perhaps is indicative of a general gender difference in global motion perception, rather than one specific to age. Snowden and Kavanagh (2006) also found gender effects on both tasks of motion coherence and speed discrimination, but none of these interacted with age. Although there is evidence that males exhibit better performance than females in tasks that involve cognitive aspects of vision such as visual navigation (e.g. Astur, Ortiz \& Sutherland, 1998) and mental rotation (Jansen \& Heil, 2010), with the exception of the findings of Billino et al. (2008) and Snowden and Kavanagh (2006), there is little evidence that this is generally the case in more low-level visual tasks such as global motion perception. The possible existence of gender-specific deficits in the processing of moving patterns and the role of sex hormones in visual cortex activity is, however, an interesting issue that certainly warrants further study.

\section{Optical factors}

It is well known that the normal aging process is accompanied by a number of changes in the optics of the eye that impact upon the quality of the visual signal prior to, or at the level of, the retina (e.g. Said \& Weale, 1959; Ruddock, 1965; Weale, 1975, 1986). Age-related reductions in pupil diameter (senile miosis) for example lead to decreased retinal illumination and increased intraocular light scatter. Reductions in the elasticity of the lens lead to decreased accommodative amplitude and presbyopia (the inability to focus on near objects). The lens also becomes denser and yellows with age, reducing transparency and consequently the quality of the retinal image produced (for a comprehensive review, see Weale 1986). It is important therefore to rule out optical factors as the cause of age-related deficits in global motion processing. 
Although optical changes inevitably lead to some degradation of the visual signal, they are insufficient to explain many of the visual deficits associated with age. Even when age-related optical factors such as lenticular yellowing and decreased pupil diameter have been accounted for, problems in spatial and temporal vision persist (e.g. Elliott, Whitaker, \& MacVeigh, 1990; Whitaker \& Elliott, 1992; Elliott, Choi, Doble, Hardy, Evans \& Werner, 2009; Allen et al., 2010). Moreover, Ball and Sekuler (1986) assessed the effects of positive optical blur $(0.00$ to +4.00 dioptres $)$ on visual acuity and global motion discrimination in young participants. They found that although blur has a deleterious effect on visual acuity, global motion perception was unaffected. It is likely therefore that age-related deficits in global motion processing reflect changes in the visual pathway beyond the retina.

\section{Neural correlates}

Important insights into the neural substrates underlying the deterioration in global motion processing can be gained by mapping specific age-related psychophysical deficits onto our current understanding of how global motion is encoded in the younger visual system. Much of our knowledge about motion processing in human visual cortex comes from studies that have investigated the neural mechanisms underlying motion processing in non-human primates. The findings of these studies suggest prominent roles for both the striate cortex (area V1) and extrastriate visual area V5/MT in global motion perception. Direction-selective neurons in V1 encode the motion direction of local elements, the outputs of which project up-stream to area V5/MT where receptive fields are much larger (Livingstone, Pack \& Born, 2001). Neurons in V5/MT integrate the local motion responses from V1 into a global representation (Figure 2), and exhibit some degree of speed-dependent tuning to translational motion (Perrone \& Thiele, 2001).

Neurophysiological evidence complements existing psychophysical evidence that translational motion perception declines with age. Schmolesky, Wang, Pu and Leventhal (2000) found that neurons in V1 of aged macaque monkeys are less direction and orientation selective than those of their younger counterparts, exhibiting increased responses to all directions and orientations and higher spontaneous activity. Liang et al. (2008) compared the proportion of directionally-selective V5/MT cells in young and old macaque monkeys and found that the proportion of such cells were lower in old animals. They also found that the degree of direction selectivity was more severely compromised in V5/MT than in V1 of senescent monkeys. The speed dependence of age-related motion deficits may reflect changes in the underlying speed tuning of visual cortical areas that respond to motion. The impairments found at relatively high speeds in particular suggest severely lowpass tuning with respect to motion, with a lower cut-off frequency in the aged. Indeed, there is neurophysiological evidence to suggest that this may be the case. Zhang, Wang, Wang, et al. (2008) have shown that neurons in area V1 of aged rhesus monkeys exhibit reduced high temporal frequency cut-offs and lower optimal temporal frequencies, compared to younger animals. Similarly Yang, Zhang, Liang et al. (2009) have provided evidence for a significant degradation of the speed selectivity of motion-sensitive cells in primate V5/MT. Cells in old animals preferred lower speeds than did those in young animals. In addition, evidence that older adults' performance is more markedly affected by local dot contrast (e.g. Allen et al. 2010) probably 
reflects contrast-dependent input from area V1 to area V5/MT, demonstrating the importance of other factors such as changes in contrast sensitivity with aging.

\section{Enhanced global motion perception in the elderly?}

Although aging typically leads to impaired performance on tasks of global motion, this is not always the case. Indeed under some circumstances older participants can exhibit superior performance relative to their younger counterparts. Hutchinson, Allen and Ledgeway (2011) have recently shown that, when stimulus area is relatively large and dot density low, younger (20 to 30 years) and older (65+ years) participants' global motion coherence thresholds are the same. However when stimulus area is relatively small $\left(\leq 57 \mathrm{deg}^{2}\right)$, younger participants' performance was markedly worse (coherence thresholds were higher) than older participants' performance. These findings suggest therefore that, in some instances, aging may lead to superior global motion processing

Enhanced motion perception in older adults has also been shown for high contrast sine-wave patterns under some conditions (Betts, Taylor, Sekuler \& Bennett, 2005). It is also of note that superior global motion perception (relative to controls) has been shown in certain patient groups such as those suffering from schizophrenia (Tadin, Kim, Doop et al., 2006) and depression (Golomb, McDavitt, Ruff et al., 2009). It has been suggested that these findings may reflect reduced centre-surround antagonism in motion processing areas such as V5/MT. Centre-surround antagonism is characteristic of some, but not all, neurons in area V5/MT (Tadin \& Blake, 2005; Aaen-Stockdale, Thompson, Huang \& Hess, 2009; Glasser \& Tadin, 2010) and refers to the reduction in neuron responsiveness when the size of a stimulus increases to the degree that it encroaches on a neuron's inhibitory surround. It is suggested to be manifest psychophysically as spatial suppression, where the ability to discriminate the direction of high contrast moving patterns becomes impaired as stimulus size increases (e.g. Tadin, Lappin, Gilroy \& Blake, 2003), particularly when the stimulus duration is brief $(<100 \mathrm{~ms}$ ). It has been proposed (e.g. Tadin \& Blake, 2005; Tadin et al., 2006; Golomb et al., 2009) that the superior global motion perception in the elderly and in a number of patient groups is due to reduced cortical inhibition leading to reduced centre-surround antagonism in V5/MT and less spatial suppression. Indeed, reduced cortical inhibition in the aging primate brain has been shown to produce improved cortical function and has been linked to age-related reductions in activity of the inhibitory neurotransmitter GABA. Leventhal, Wang, Pu, Zhou and Ma (2003) for example found that the application of GABA led to significant narrowing of direction and orientation tuning in neurons in area V1 of the macaque. Neurons fired almost exclusively to their preferred direction and/or orientation. Similar results were obtained in the presence of the $\mathrm{GABA}_{\mathrm{A}}$ agonist muscimol. Application of GABA and muscimol also led to decreased visual responsiveness, decreased spontaneous activity, and increased signal-to-noise ratios. Conversely, the application of $\mathrm{GABA}_{\mathrm{A}}$ antagonist bicuculline produced a non-specific increase in neuron firing rate (increased peak responses and spontaneous activity, and decreased signal-to-noise ratios). Added support for the inhibitory account comes from a recent study (Tadin, Silvanto, Pascual-Leone \& Battelli, 2011) that showed that disrupting human area V5/MT with transcranial magnetic stimulation (TMS) led to improved motion discrimination performance for high contrast moving patterns, a finding the authors propose is due to TMS-induced weakening of surround suppression strength. 


\section{More complex types of global motion}

The overwhelming majority of studies that have investigated the effects of age on global motion perception have done so using patterns in which elements move along a translational trajectory. Many of their findings have been taken as evidence of agerelated changes in area V5/MT. Relatively few studies have used RDK patterns in which dots move along more complex 'optic flow' trajectories (see Figure 3) and the results have been mixed for those that have. For example Atchley and Anderson (1991) and Billino et al., (2008) found no differences in the perception of radial global motion with increasing age. Conversely, Mapstone, Dickerson and Duffy (2008) found some evidence of an age-related decline and this may be indicative of changes in other parts of the motion-processing pathway beyond V5/MT. Other studies (e.g. Allen et al., 2010) found age-related declines for radial and rotational global motion but only when the dot contrast was low and close to its own detection threshold. It is likely however that these particular findings reflect age-related changes in contrast sensitivity, rather than in global motion perception per se. The neurophysiological correlates of age-related deficits in more complex types of global motion (e.g. rotational and radial motion) are likely to lie outside area V5/MT, where neurons respond to even more complex features of a global motion stimulus. In adjacent area MST, for example, neurons respond selectively to radial and circular motion (Duffy \& Wurtz, 1991) such as expanding and contracting movements, rotations or even spiraling motions and has been proposed as a prime candidate for encoding the patterns of optic flow generated by self-motion during visually-guided navigation (Grossberg, Mingolla \& Pack, 1999). As such, deficits in radial or rotational motion are likely to reflect changes in higher motion-processing areas such as MST.

The effects of age on the perception of global motion patterns in which dots are defined by characteristics other than luminance have yet to be examined. Secondorder, contrast-defined global motion patterns for example require more complex levels of analysis by the visual system than their first-order, luminance-defined counterparts. On that basis, it has often been proposed that second-order motion processing relies on separate, and perhaps 'higher order' extrastriate brain areas (e.g. Baker, 1999; Vaina \& Soloviev, 2004). In the context of amblyopia for example, Simmers, Ledgeway, Hess and McGraw (2003) have shown greater deficits for second-order (contrast-defined) global motion processing than for first-order (luminance-defined) global motion processing, relative to a 'normal' population. In the context of aging, Tang and Zhou (2009) found that modulation sensitivity for discriminating the direction of second-order grating patterns declined earlier in the aging process than contrast sensitivity for discriminating the direction of first-order grating patterns. They attributed this earlier decline for second-order, compared to first-order, stimuli to the greater complexity involved in second-order processing. Future studies examining the effects of aging on second-order global motion perception should therefore refine our understanding of age-related changes in extrastriate visual areas.

\section{Summary and future considerations}


Evidence suggests that the normal aging process selectively impairs translational global motion perception. This is certainly true in those over the age of 70 years and there may some degree of more graded impairments throughout the lifespan. Older adults are particularly impaired when local elements move at very high (or low) speeds, are displaced over relatively large spatial extents, or are low in contrast. Under some conditions (i.e. intermediate speeds and displacements, large display areas), younger and older participants appear to exhibit equivalent performance. In others (i.e. relatively small display regions, high dot densities), older adults may even exhibit better performance. There is also some limited evidence to suggest that any detrimental effects of age on global motion perception are more marked in older females.

Identifying the precise nature of the visual changes associated with normal aging using behavioural (psychophysical) measures is important for understanding their consequences in the real world. Evidence from other neuroscientific techniques such as fMRI are also likely to provide useful insights into their neural origins. It is important however that research also looks towards therapeutic interventions that will alleviate the practical problems experienced by older adults as a result of their visual deficits. One avenue for potentially improving vision in the elderly is perceptual learning. Visual perceptual learning is a well-established phenomenon in normal vision and occurs when perceptual training on a task leads to improved visual performance (e.g. Ball \& Sekuler, 1987; Gold, Bennett \& Sekuler, 1999; Sigman \& Gilbert, 2002). The classical notion of perceptual learning was that it was specific to childhood, diminishing dramatically with age. However, it is now clear that there exists marked plasticity in the adult visual system where learning has been demonstrated across a wide range of visual tasks including orientation, motion, texture, shape and face discrimination (see Fine \& Jacobs, 2002; Sagi, 2011 for reviews). Evidence suggests that perceptual learning may also be effective in older adults, although surprisingly few studies have examined this issue. Ball and Sekuler (1986) demonstrated that practice on a translational global motion discrimination task led to improved performance in older adults (62 to 72 years). Moreover, older adults showed the same rate of practice-related improvement as their younger counterparts. Andersen, Ni, Bower, and Watanabe (2010) measured texture discrimination performance and found that older participants (mean age 72 years) were markedly impaired compared to younger participants (mean age 21 years). They subsequently trained the older individuals on the task and found that, after only two days training, older participants' performance had improved and matched the young participants' pre-training performance level. These promising findings may also hold for other visual tasks such as those that involve the extraction of a global motion signal. As such, perceptual learning may prove to be a useful tool for improving vision-related quality of life in the elderly.

In conclusion, the normal aging process appears to differentially compromise some, but not all, aspects of global motion perception. Translational motion perception may be particularly susceptible to age-related deficits but more research using other types of global motion (e.g. radial \& rotational patterns) is necessary before any firm conclusions can be drawn in this respect. Many of the psychophysical deficits reported cannot be accounted for by age-related optical factors and, as a consequence, are likely to reflect changes in the cortex. In some studies there is evidence of a gender bias, with women appearing to be more markedly affected by age-related 
changes than men. Future work should aim to provide a clearer picture of the precise psychophysical deficits associated with age for all global motion types, including those defined by second-order properties such as contrast or texture, with a view to uncovering the underlying mechanisms that lead to such profound changes in perception. Another promising avenue for future research concerns examining the efficacy of perceptual learning paradigms with a view to developing therapeutic interventions that could potentially improve global motion perception in the elderly.

\section{References}

Aaen-Stockdale, C.R., Thompson, B., Huang, P.C. \& Hess, R.F. (2009). Low-level mechanisms may contribute to paradoxical motion percepts. Journal of Vision, 9(5):9, $1-14$.

Allen, H.A., Hutchinson, C.V., Ledgeway, T. and Gayle, P. (2010). The role of contrast sensitivity in global motion processing deficits in the elderly. Journal of Vision, 10(10):15, 1-10.

Andersen, G. J., \& Ni, R. (2008). Aging and visual processing: Declines in spatial not temporal integration. Vision Research, 48(1), 109-118.

Andersen, G.J., Ni, R., Bower, J.D., and Watanabe, T. (2010). Perceptual learning, aging, and improved visual performance in early stages of visual processing. Journal of Vision, 10(13):4, 1-13.

Astur, R.S., Ortiz, M.L. \& Sutherland, R.J. (1998). A characterization of sex performance by men and women in a virtual Morris water task: A large and reliable sex difference. Behavioural Brain Research, 93, 185-190.

Atchley, P. \& Andersen, G. J. (1998). The effect of age, retinal eccentricity, and speed on the detection of optic flow components Psychology and Aging 13(2) 297308.

Arena, A., Hutchinson, C.V. and Shimozaki, S.S. (2011). Speed-dependent motion processing deficits in aging: The role of spatial integration. Perception (Supplement), $40,114$.

Baker, C.L. Jr. (1999). Central neural mechanisms for detecting second-order motion. Current Opinion in Neurobiology, 9, 461-466.

Ball, K. \& Sekuler, R. (1986). Improving visual perception in older observers. Journal of Gerontology, 41(2), 176-182.

Ball, K., \& Sekuler, R. (1987). Direction-specific improvement in motion discrimination. Vision Research, 27, 953-965.

Bennett, P. J., Sekuler, R., \& Sekuler, A. B. (2007). The effects of aging on motion detection and direction identification. Vision Research, 47(6), 799-809. 
Betts, L.R., Taylor, C.P., Sekuler, A.B., Bennett, P.J. (2005). Aging reduces center surround antagonism in visual motion processing. Neuron, 45, 361-366.

Billino, J., Bremmer, F., \& Gegenfurtner, K. R. (2008). Differential aging of motion processing mechanisms: Evidence against general perceptual decline. Vision Research, 48(10), 1254-1261.

Duffy, C.J. \& Wurtz, R.H. (1991). Sensitivity of MST neurons to optic flow stimuli I. A continuum of response selectivity to large-field stimuli. Journal of Neurophysiology, 65, 1329-1345.

Elliott, D.B., Whitaker, D. \& MacVeigh, D. (1990). Neural contribution to spatiotemporal contrast sensitivity decline in healthy eyes. Vision Research, 30, 541547.

Elliott, S.L., Choi, S.S., Doble, N., Hardy, J.L., Evans, J.W. \& Werner, J.S. (2009). Role of higher-order aberrations in senescent changes in spatial vision. Journal of Vision, 9, 1-16.

Fine, I. \& Jacobs, R.A. (2002). Comparing perceptual learning across tasks: A review. Journal of Vision, 2, 190-203.

Hutchinson, C.V., Allen, H.A. and Ledgeway, T. (2011). When older is better:

Superior global motion perception in the elderly. Perception (Supplement), 40, 115.

Gilmore, G. C., Wenk, H. E., Naylor, L. A., \& Stuve, T. A. (1992). Motion perception and aging. Psychology and Aging, 7(4), 654-660.

Glasser, D.M. \& Tadin, D. (2010). Low-level mechanisms do nto explain paradoxical motion percepts. Journal of Vision, 10(4):20. 1-9.

Gold, J., Bennett, P. J., \& Sekuler, A. B. (1999). Signal but not noise changes with perceptual learning. Nature, 402, 176-178.

Golomb, J.D., McDavitt, J.R., Ruf, B.M., Chen, J.I., Saricicek, A., Maloney, K.H., Hu, J.,Chun, M.M. \& Bhagwagar, Z. (2009), Enhanced visual motion perception in major depressive disorder. Journal of Neuroscience, 29, 9072-9077.

Grossberg, S., Mingolla, E. \& Pack, C. (1999). A neural model for motion processing and visual navigation by cortical area MST. Cerebral Cortex, 9, 878-895.

Jansen, P. \& Heil, M. (2010). Gender differences in mental rotation across adulthood. Experimental Aging Research, 36, 94-104.

Leventhal, A.G., Wang, Y.C., Pu, M.L., Zhou, Y.F. \& Ma, Y.Y. (2003). GABA and its agonists improved visual cortical function in senescent monkeys. Science, 300, 812-815.

Livingstone, M.S., Pack, C.C. \& Born, R.T. (2001). Two-dimensional substructure of MT receptive fields. Neuron, 30, 781-793. 
Mapstone, M., Dickerson, K. \& Duffy, C.J. (2008). Distinct mechanisms of impairment in cognitive ageing and Alzheimer's disease. Brain, 131, 1618-1629.

Newsome, W.T. \& Paré, E.B. (1988). A selective impairment of motion perception following lesions of the middle temporal visual area (MT). The Journal of Neuroscience, 8, 2201-2211.

Perrone, J.A, \& Thiele A. (2001). Speed skills: measuring the visual speed analyzing properties of primate MT neurons. Nature Neuroscience, 4, 526-532.

Roudaia, E., Bennett, P., Sekuler, A., \& Pilz, K. S. (2010). Spatiotemporal properties of apparent motion perception and aging. Journal of Vision, 10(14), 1-15.

Ruddock K. H. (1965). The effect of age upon colour vision II. Changes with age in light transmission of the ocular media Vision Research, 5, 47-58.

Sagi, D. (2011). Perceptual learning in Vision Research. Vision Research, 51, 15521566.

Schmolesky, M. T., Wang, Y. C., Pu, M. L., \& Leventhal, A. G. (2000). Degradation of stimulus selectivity of visual cortical cells in senescent rhesus monkeys. Nature Neuroscience, 3, 384-390.

Sigman, M., \& Gilbert, C. D. (2000). Learning to find a shape. Nature Neuroscience, 3, 264-269.

Simmers, A.J., Ledgeway, T., Hess, R.F. And McGraw, P.V. (2003). Deficits to global motion processing in human amblyopia. Vision Research, 43, 729-738.

Smith, A. T., Snowden, R. J., \& Milne, A. B. (1994). Is global motion really based on spatial integration of local motion signals? Vision Research, 34(18), 2425-2430.

Snowden, R. J., \& Kavanagh, E. (2006). Motion perception and the ageing visual system: Minimum motion, motion coherence, and speed discrimination thresholds. Perception, 35(1), 9-24.

Tadin, D. \& Blake, R (2005) Motion perception getting better with age? Neuron, 45, $325-327$.

Tadin, D., Kim, J., Doop, M.L., Gibson, C., Lappin, J.S., Blake, R. and Park, S. (2006). Weakened center-surround interactions in visual motion processing in schizophrenia. Journal of Neuroscience, 2, 11403-11412.

Tadin, D., Lappin, J.S., Gilroy, L.A., Blake, R. (2003). Perceptual consequences of centre-surround antagonism in visual motion processing. Nature, 424, 312-315.

Tadin, D., Silvanto, J., Pascual-Leone, A. \& Batelli, L. (2011), Improved motion perception and impaired spatial suppression following disruption of cortical area MT/V5. Journal of Neuroscience, 31, 1279-1283. 
Tang, Y. and Zhou, Y-F. (2009). Age-related decline of contrast sensitivity for second-order stimuli: Earlier onset but slower progression, than for first-order stimuli. Journal of Vision, 9(7):18, 1-15.

Tran D. B., Silverman, S. E., Zimmerman, K., \& Feldon, S. E. (1998). Age-related deterioration of motion perception and detection. Graefe's Arch Clin Exp Ophthamology, 236, 269-273.

Trick, G. L., \& Silverman, S. E. (1991). Visual sensitivity to motion: age-related changes and deficits in senile dementia of the Alzheimer type. Neurology, 41, 14371440 .

Vaina, L.M. \& Soloviev, S. (2004). First-order and second-order motion: neurological evidence for neuroanatomically distinct systems. Progress in Brain Research, 144, 197-212.

Weale R. A. (1975). Senile changes in visual acuity. Transactions of the Ophthalmological Society UK, 95, 36-38.

Weale, R.A. (1986). Aging and vision. Vision Research, 26, 1507-1512.

Whitaker, D., \& Elliott, D. B. (1992) Simulating age-related optical changes in the human eye. Documenta Ophthalmologica, 82, 307-316.

Wojciechowski, R., Trick, G. L., \& Steinman, S. B. (1995). Topography of the agerelated decline in motion sensitivity. Optometry and Vision Science, 72(2), 67-74.

Yang, Y., Zhang, J., Liang, Z., Li, G., Wang, Y., Ma, Y., Zhou, Z. \& Leventhal, A.G. (2009). Aging effects the neural representation of speed in macaque area MT.

Cerebral Cortex, 19, 1957-1967.

Zhang, J., Wang, X., Wang, Y. Fu, Y., Liang, Z., Yuanye, M. \& Leventhal, A.G. (2008). Spatial and temporal sensitivity degradation of primary visual cortical cells in senescent rhesus monkeys. European Journal of Neuroscience, 28, 201-207.

Zihl, J., D. Von Cramon, and N. Mai (1983) Selective disturbance of movement vision after bilateral brain damage. Brain, 106: 313-340. 
Figure

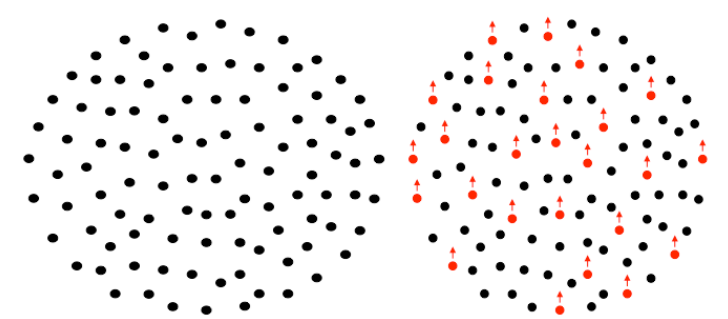

A: $0 \%$ motion coherence

B: $25 \%$ motion coherence

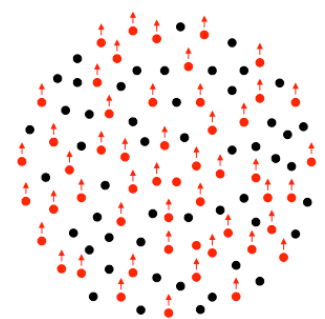

C: $\mathbf{5 0} \%$ motion coherence

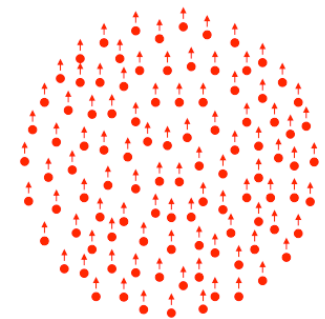

D: $100 \%$ motion coherence

Figure 1. Schematic example of an RDK stimulus depicting translational motion.

Signal dots are depicted with arrows. Noise dots are depicted without arrows but in reality move in random directions. A: All of the dots are noise dots moving in random directions, producing a motion coherence level of $0 \%$. B: $25 \%$ of the local dots are signal dots moving in a common direction (in this case upwards) and $75 \%$ are noise dots moving randomly, producing a motion coherence level of $25 \%$. C: $50 \%$ of the dots are signal dots and $50 \%$ are noise dots, producing a motion coherence level of $50 \%$. D: The display contains only signal dots moving upwards, producing a motion coherence level of $100 \%$.

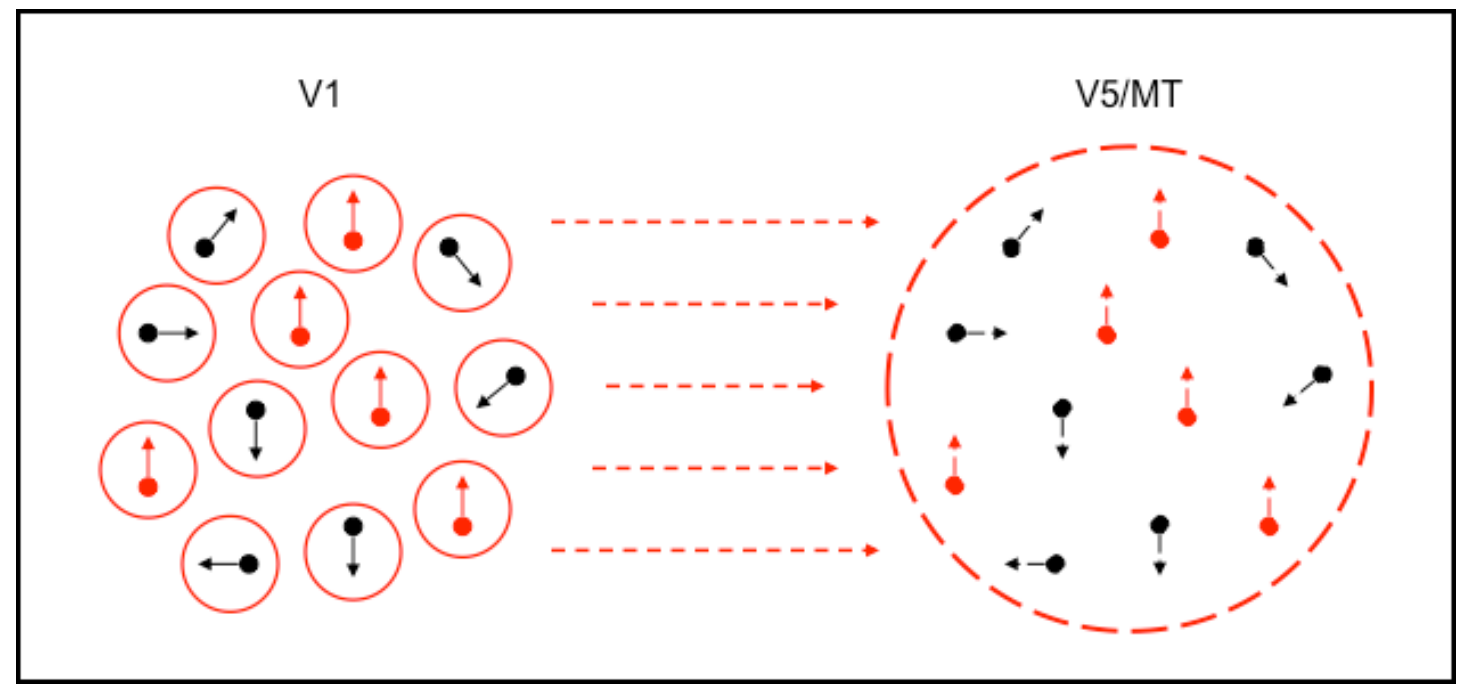

Figure 2. Direction-selective neurons in area V1 have relatively small, spatially localised receptive fields (e.g. $<1 \mathrm{deg}$ in the fovea). As such, each is only able to encode the motion of individual dots within a restricted spatial area of the image. This means that the output of any individual neuron is ambiguous because the motion measured within any local receptive fields is not necessarily indicative of the overall (global) image motion. This is referred to as the aperture problem and is resolved because the output of V1 neurons project up-stream to extrastriate area V5/MT where neurons have much larger receptive fields. V5/MT provides a second stage of motion analysis whereby the responses of V1 neurons are integrated (combined, compared or pooled) to determine the global direction and speed. 

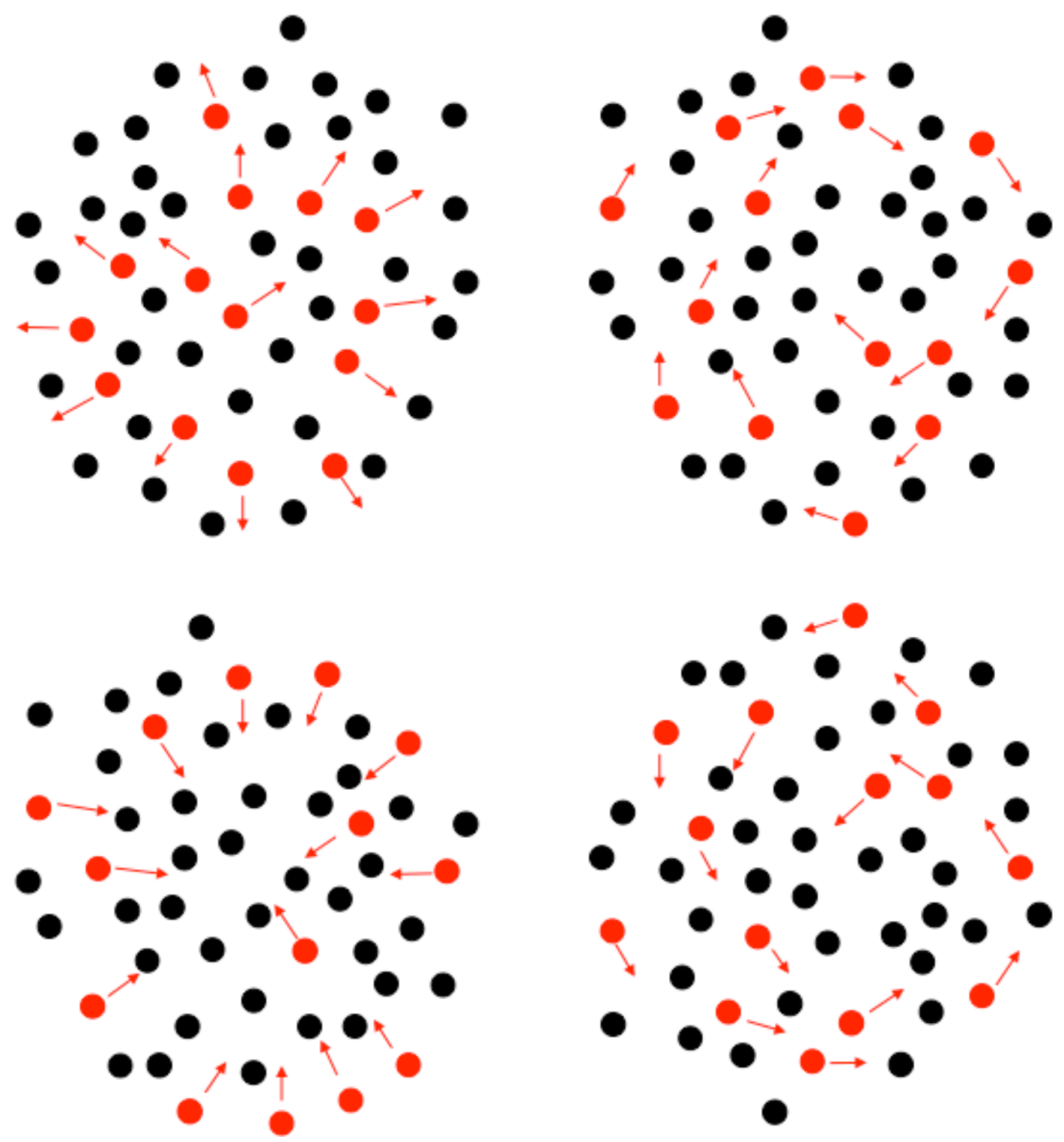

Figure 3. Examples of radial and rotation motion. A: Radial motion. Signal dots move along trajectories that depict either expansion (top) or contraction (bottom). B: Rotational motion. Signal dots rotate either clockwise (top) or anti-clockwise (bottom). Noise dots always move in random directions spanning the 360 deg range. 
Table 1. A synopsis of the speed-dependence of translational global motion perception in the elderly.

\begin{tabular}{|c|c|c|c|}
\hline Study & $\begin{array}{l}\text { Local } \\
\text { dots } \\
\text { speed } \\
(\text { deg/sec })\end{array}$ & $\begin{array}{l}\text { Difference } \\
\text { between } \\
\text { young and } \\
\text { old? }\end{array}$ & Points of note \\
\hline $\begin{array}{l}\text { Ball \& Sekuler } \\
(1986)\end{array}$ & 10 & YES & \\
\hline $\begin{array}{l}\text { Trick \& } \\
\text { Silverman } \\
\text { (1991) }\end{array}$ & 5.8 & YES & \\
\hline $\begin{array}{l}\text { Gilmore et al. } \\
\text { (1992) }\end{array}$ & 3.9 & $\mathrm{NO}$ & $\begin{array}{l}\text { No overall difference between younger and } \\
\text { older observers, but some of the elderly female } \\
\text { participants were marginally more impaired }\end{array}$ \\
\hline $\begin{array}{l}\text { Wojciechowski } \\
\text { et al. (1995) }\end{array}$ & 28 & YES & \\
\hline $\begin{array}{l}\text { Atchley \& } \\
\text { Andersen (1998) }\end{array}$ & $4.8 \& 22$ & YES & $\begin{array}{l}\text { At } 4.8 \mathrm{deg} / \mathrm{sec} \text {, only older women were } \\
\text { impaired. This was only the case in central } \\
\text { vision. } \\
\text { At } 22 \mathrm{deg} / \mathrm{sec} \text {, older participants were impaired } \\
\text { relative to younger participants, except at a } \\
\text { viewing eccentricity of } 40 \mathrm{deg} \text {, where young } \\
\text { and old participants' performance was } \\
\text { equivalent. }\end{array}$ \\
\hline $\begin{array}{l}\text { Tran et al. } \\
(1998)\end{array}$ & 2.5 & YES & \\
\hline $\begin{array}{l}\text { Snowden \& } \\
\text { Kavanagh } \\
\text { (2006) }\end{array}$ & $0.045-4$ & YES & $\begin{array}{l}\text { Age-related deficits only evident at speeds } \leq 1 \\
\text { deg/sec. }\end{array}$ \\
\hline $\begin{array}{l}\text { Bennett et al. } \\
(2007)\end{array}$ & 6 & YES & \\
\hline $\begin{array}{l}\text { Billino et al. } \\
(2008)\end{array}$ & $\begin{array}{l}6.6 \& \\
18.6 \\
\end{array}$ & YES & $\begin{array}{l}\text { Age-related deficits apparent for both dot } \\
\text { speeds }\end{array}$ \\
\hline $\begin{array}{l}\text { Allen et al. } \\
(2010)\end{array}$ & 5.6 & YES & Age-related deficits only at low dots contrasts \\
\hline
\end{tabular}

